Ähnliche Tendenzen zeigen sich auch im deutschsprachigen Raum; so beschreibt sich die deutsche Epoch Times als «frei von Propaganda und Medienzensur»" das österreichische Online-Medium Unzensuriert verweist auf «Fake News» öffentlich-rechtlicher Medien ${ }^{2}$ und die Schweizer Website Alles Schall und Rauch versucht, eine Verschwörung hinter den Terroranschlägen des 11. September aufzudecken ${ }^{3}$. Gleichzeitig etablieren sich im DACH-Raum seit einigen Jahren Nachrichtenseiten, die für vertiefte Recherchen und Hintergrundberichterstattung einstehen und sich von der Kommerzialisierung des Mainstream-Mediensystems abzugrenzen versuchen, so beispielsweise die deutsche Nachrichtenseite Krautreporter, die Schweizer Republik oder das österreichische Online-Magazin Addendum. Die Schwierigkeit, den Begriff «Alternativmedien» in digitalen Öffentlichkeiten zu fassen, verdeutlichen Beispiele wie diese, die auf den ersten Blick unterschiedliche Ausrichtungen und Ziele zu verfolgen scheinen. Im Rahmen der vorliegenden Arbeit soll daher das Phänomen alternativer Nachrichtenmedien in Deutschland, Österreich und der deutschsprachigen Schweiz (dem DACH-Raum) explorativ untersucht werden. Alternative Nachrichtenmedien werden in diesem Kontext als eine mögliche Form von Gegenöffentlichkeit und als Gegenpol zur massenmedial hergestellten Öffentlichkeit durch professionelle Nachrichtenmedien betrachtet. Die Begriffe «Alternativmedien» und «alternative Nachrichtenmedien» werden in weiterer Folge synonym verwendet, zumal in der bisherigen wissenschaftlichen Literatur vor allem der erste Begriff tragend ist. Dennoch wird der Begriff «alternative Nachrichtenmedien» für den Fokus dieser Arbeit bevorzugt, zumal die empirische Untersuchung auf Nachrichtenmedien fokussiert, die regelmässig Beiträge mit Aktualitätswert verbreiten. Der Fokus auf den DACH-Raum ist nicht nur durch den diesbezüglich vorhandenen Forschungsbedarf begründet, sondern vor allem durch die Annahme, dass alternative Nachrichtenmedien im digitalen Raum länderübergreifend agieren und im gesamten deutschsprachigen Raum vernetzt agieren (Vogler, 2017). Die konkrete Problem- und Fragestellung wird folgend erläutert.

\title{
I.1 Problemstellung
}

Die von Medien ausgehende Kritik- und Kontrollfunktion gegenüber politischen Machtträger_innen ist unzweifelhaft eine wesentliche Funktion öffentlicher Kommunikation. Medien als sogenannte Vierte Gewalt neben Legislative, Exeku-

\footnotetext{
1 https://www.epochtimes.de/thema/epoch-times/epoch-times-epochtimes-a4717.html (Stand: 14.11.2018)

2 https://www.unzensuriert.at/?s=fake+news (Stand: 14.11.2018)

3 http://alles-schallundrauch.blogspot.com (Stand: 16.11.2020)
} 
tive und Judikative informieren die Gesellschaft über das politische Geschehen und können dadurch gegebenenfalls auch Missstände aufdecken. Als etablierte Medien bezeichnete Massenmedien haben dahingehend lange Zeit eine Gatekeeping-Funktion eingenommen (vgl. hierzu auch Wallace, 2017), selektierten und kontrollierten Informationen, die dann in der Öffentlichkeit verbreitet wurden. Dieser Prozess hat sich durch die Digitalisierung stark gewandelt, da es über Plattformen auch Lai_innen möglich ist, öffentlich Nachrichten aufzubereiten und zu verbreiten (Eisenegger, 2017; Neuberger \& Quandt, 2010; Nuernbergk, 2012; Wallace, 2017). Die vorliegende Arbeit fokussiert auf ebendiese neuen öffentlichen Kommunikator_innen und stellt die Frage, inwiefern sich durch den digitalen Wandel potentielle Gegenöffentlichkeiten ausformieren, etablieren und ihre Netzwerke online aufbauen. Unter Gegenöffentlichkeit wird «eine gegen eine hegemoniale Öffentlichkeit gerichtete Teilöffentlichkeit [verstanden], die um einen spezifischen gesellschaftlichen Diskurs oder Standpunkt herum strukturiert ist» (Krotz, 1998, S. 653). Demzufolge liegt der Fokus dieser Studie auf gesellschaftlichen Gruppen, die sich gegen den (häufig von diesen Gruppen selbst verwendeten und negativ konnotierten Begriff) «Mainstream» positionieren, der die öffentliche Meinung vorzugeben scheint. Gegenöffentlichkeiten richten sich demnach gegen politische, aber auch mediale Eliten und deren Äusserungen. Die vorliegende Arbeit interessiert sich konkret für Gegenöffentlichkeiten im digitalen Zeitalter und widmet sich in diesem Kontext dem Phänomen alternativer Nachrichtenmedien. Obwohl alternative Nachrichtenmedien ihre Ursprünge beispielsweise in der politisch links positionierten Alternativpresse der 1960 und $70 e r$ Jahre haben, fokussiert die aktuelle Forschung vorwiegend rechtspopulistische oder desinformative Medien, die unter den Titel «Alternativmedien» oder «alternative Nachrichtenmedien» subsumiert und als potentielle Gefahr für die demokratische Ordnung gedeutet werden. Fraglich ist allerdings, ob in der zunehmend pluralen Medienöffentlichkeit eine dahingehende einschränkende Einordnung alternativer Nachrichtenmedien ausreichend ist. Aus diesem Grund wird in dieser Studie eine differenziertere Betrachtung alternativer Medien angestrebt und basierend auf einem phänomenologischen Ansatz erstmals das aktuelle Feld alternativer Online-Nachrichtenmedien im deutschsprachigen Raum analysiert und definiert. Folgende übergeordnete Fragestellung ist für die vorliegende Studie forschungsleitend:

Wie lassen sich Gegenöffentlichkeiten wie alternative Online-Nachrichtenmedien in Deutschland, Österreich und der deutschsprachigen Schweiz definitorisch einordnen und welchen Stellenwert nehmen sie in der öffentlichen Kommunikation auf digitalen Plattformen ein? 
Neben einer theoretischen Einordnung der Öffentlichkeits- und Gegenöffentlichkeitsbegriffe wird das Thema empirisch anhand von vier Forschungsfragen bearbeitet. Dabei wird im Rahmen von Forschungsfrage 1 untersucht, welche alternativen Nachrichtenmedien im DACH-Raum aktuell online via Website und Accounts auf Digitalplattformen agieren. Forschungsfrage 2 legt den Fokus auf das Selbstverständnis alternativer Nachrichtenmedien, um diese zu definieren und typologisch einzuordnen. Forschungsfrage 3 widmet sich schliesslich einer netzwerkanalytischen Betrachtung alternativer und professioneller deutschsprachiger Nachrichtenmedien auf der Plattform Twitter. Dieser deskriptive Zwischenschritt ist massgeblich für die Beantwortung von Forschungsfrage 4, im Zuge derer jene Netzwerkrelationen interpretativ analysiert werden.

\section{I.2 Relevanz}

Was passiert, wenn sich in Zeiten digitaler Kommunikation der erweiterte Kreis an Kommunikator_innen oder «pseudojournalistischen» Anbietern (Eisenegger, 2017) nicht an journalistische Standards, wie beispielsweise Quellentransparenz oder Objektivität, halten? Wenn desinformative, verschwörungstheoretische Beiträge öffentlich zirkulieren? Oder politisch gefärbte, polarisierende Meinungen und Nachrichten verbreitet werden? Die Auseinandersetzung mit alternativen Nachrichtenmedien ruft ebensolche Fragen hervor. Die Relevanz, alternative Nachrichtenmedien im deutschsprachigen Raum $\mathrm{zu}$ untersuchen, ergibt sich unbestreitbar aufgrund potentieller Gefahren für die soziale Ordnung respektive Demokratie. Die von der Etablierung digitaler Plattformen geprägte digitale Öffentlichkeit bietet aufgrund ihres partizipativen Charakters den idealen Nährboden für alternative Kommunikator_innen. Ein auf Vernunft und Argumenten basierter Diskurs als Basis unserer Demokratie ist bei alternativen Kommunikator_innen aber gerade dann in Frage zu stellen, wenn absichtlich Sachverhalte verdreht, Falschnachrichten verbreitet, oder Gruppen diffamiert werden. Ausserdem impliziert der Begriff «Alternativmedium» eine relationale Abgrenzung zu klassischen Medien. Sie positionieren sich gegen die mediale und politische Öffentlichkeit, wodurch einerseits die Gefahr besteht, dass sich Publika von Alternativmedien ein Bild der Wirklichkeit in abgeschlossenen Teil- respektive Gegenöffentlichkeiten machen und mit professionellen journalistischen Beiträgen kaum oder gar nicht in Berührung kommen. Darüber hinaus können alternative Nachrichtenmedien eine Polarisierung der Gesellschaft weiter fördern - im Sinne von: Wir gegen die anderen. Nichtsdestoweniger erscheint es wesentlich, das Spektrum alternativer Nachrichtenmedien genauer unter die Lupe zu nehmen. Eine dichotome Unterscheidung in Öffentlichkeit und Gegenöffentlichkeit - respektive: professionelle und alternative Nachrichtenmedien - erscheint vor allem 